\title{
A New Agonist for Peroxisome Proliferation- activated Receptor $\gamma$ (PPAR $\gamma$ ), Fraglide-1 from Zhenjiang Fragrant Vinegar: Screening and Characterization Based on Cell Culture Experiments
}

\author{
Yoshio Tsujino* \\ School of Materials Science, Japan Advanced Institute of Science and Technology, 1-1 Asahidai, Nomi, Ishikawa 923-1292, JAPAN
}

\begin{abstract}
Zhenjiang fragrant vinegar (Kozu) is a black rice vinegar that has been used as a traditional Chinese medicine and has various health benefits, including anti-obesity effects. In the present study, using luciferase reporter assays of PPAR $\gamma$ promoter activity, a novel ingredient from 8-year-old Kozu, 5-hydroxy4-phenyl-butenolide, was isolated. The newly found agonist was named as "Fraglide-1". Moreover, in subsequent experiments, it was confirmed that fraglide-1 was an PPAR $\gamma$ agonist and it could increase expression level of the uncoupling protein (UCP)-1. Fraglide-1 was chemically synthesized and it was verified that expression of the PPAR $\gamma$ was increased in dose dependent manner. Although Kozu has been consumed globally as a functional food for thousands of years, the mechanisms behind its health effects have not been characterized. The active ingredient of Kozu was successfully found and the results unraveled a longtime mystery about Kozu for its beneficial health effect.
\end{abstract}

Key words: fragrant vinegar, 5-hydroxy-4-phenyl-butenolide, fraglide-1, Kozu, PPAR $\gamma, \mathrm{UCP}-1$, troglitazone

\section{INTRODUCTION}

Fragrant vinegars (aromatic vinegars) are typically produced by the fermentation of glutinous rice followed by a long ageing time, and have been used for thousands of years as traditional Chinese vinegar seasonings ${ }^{1)}$. Recently, fragrant vinegars have become increasingly popular health foods globally. Whereas common vinegars are produced using 1-3-month fermentation and maturation processes, the 8-year-old hengshun fragrant vinegar (Zhenjiang Xiang Cu; Kozu) from Zhenjiang Jiangsu, China, is matured and fermented for 6 months to several years. Kozu is often marketed in a concentrated form with a darkbrown or reddish-brown color and is supplied to 160 countries as a basic flavoring material for Chinese cuisine, which is the world's most common food category. As a traditional Chinese medicine (Kampo), fragrant vinegar has long been considered as a functional food that ameliorates obesity and lifestyle-related diseases, and is described in the Chinese pharmaceutical magazine Honzo Komoku as follows: "only rice vinegar that has been aged for more than 2-3 years can be used as a medicine material.' However, although the health benefits of vinegar have been demonstrated $^{2)}$, the active ingredients of vinegars such as Kozu remain unidentified, and their effects have not been demonstrated in animal or clinical studies.

Excessive caloric intake and decreased physical activity lead to obesity, which has become a major global health issue as the main cause of lifestyle-related diseases such as diabetes, hypertension, and dyslipidemia ${ }^{3)}$. Although various medications have been developed for the treatment of metabolic abnormalities, prophylactic approaches remain essential for avoiding obesity. However, despite the increasing demand for obesity-related functional foods, the health care burden of obesity continues to increase globally, suggesting that conventional and established functional foods are not completely effective and fail to meet consumer requirements.

Currently available agents for the treatment of obesity and associated sequela include the peroxisome proliferator-activated receptor $\gamma(\mathrm{PPAR} \gamma)$ agonist, which has been widely used in the treatment of diabetes. High expression of PPAR $\gamma$ has been associated with metabolic syndrome, obesity $^{4)}$, diabetes mellitus ${ }^{5-8)}$, hypertension, arteriosclero$\mathrm{sis}^{9)}$, hyperlipidemia, inflammatory disease, and malignan-

*Correspondence to: Yoshio Tsujino, School of Materials Science, Japan Advanced Institute of Science and Technology, 1-1 Asahidai, Nomi, Ishikawa 923-1292, JAPAN

E-mail: ytsujino@jaist.ac.jp

Accepted February 3, 2017 (received for review December 31, 2016)

Journal of Oleo Science ISSN 1345-8957 print / ISSN 1347-3352 online

http://www.jstage.jst.go.jp/browse/jos/ http://mc.manusriptcentral.com/jjocs 
$\mathrm{cy}^{10)}$. Binding of agonist to PPAR $\gamma$ improves carbohydrate and lipid metabolism ${ }^{11)}$, in part reflecting the induction of mitochondrial uncoupling proteins (UCP) such as UCP-1 ${ }^{4)}$.

Although thiazolidine ingredients has demonstrated efficacy in the treatment of diabetes, it causes weight gain, fluid retention, and cardiovascular dysfunction ${ }^{6,11}$, warranting the development of dietary PPAR $\gamma$ agonists with fewer side effects. Thus, in the present study, the novel ingredient, 5-hydroxy-4-phenyl-butenolide (fraglide-1), was identified in 8-year-old Kozu (hengshun fragrant vinegar) by serially isolating active chromatographic fractions using PPAR $\gamma$ activation assays. The present observations may lead to the development of fraglide-1 containing food products that ameliorate obesity.

\section{EXPERIMENTAL}

\subsection{Fraglide-1 extraction from vinegar}

Kozu and $n$-hexane (1L each) were mixed and centrifuged $(3,400 \times g, 10 \mathrm{~min})$ to recover an aqueous layer. Phase partitioning was performed thrice. Redundant lipid components were removed. Equal volumes of chloroform were added to partitioned aqueous layers and were mixed and centrifuged to yield a chloroform layer. Recovered chloroform layers were then concentrated under reduced pressure, yielding $3970 \mathrm{mg}$ of chloroform extract.

\subsection{Normal-phase silica gel column treatment}

Chloroform extract (3970 mg) was dissolved in acetone and was adsorbed onto $130 \mathrm{~g}$ of silica gel (silica gel 60, 0.040-0.063-mm column chromatography; Merck Millipore), which was packed into a chromatographic glass column (length, $60 \mathrm{~cm}$; diameter, $2.7 \mathrm{~cm}$; volume, $343 \mathrm{~mL}$ ). Solvent mixtures $(550 \mathrm{~mL})$ with benzene:acetone at ratios of $20: 1,10: 1,5: 1,3: 1,2: 1,1: 1$ and $0: 1(\mathrm{v} / \mathrm{v})$ were added to the column in that order, and eluted fractions were collected for each solvent mixture as normal-phase column fractions (fractions 1-7). Recovered fractions were concentrated under reduced pressure using a Diaphragm vacuum pump (DIVAC 1.2L, Leybold GmbH), and residue solid weights were recorded. Portions of dried fractions were dissolved in dimethyl sulfoxide(DMSO; Nacalai Tesque, Inc., Kyoto, Japan) to $\geq 1000$ times the final concentration and were then subjected to PPAR $\gamma$ ligand activity assays.

\subsection{Cell culture}

African green monkey kidney-derived CV-1 cells or C3H10T1/2 cells (Dainippon Sumitomo Pharma, Osaka, Japan) purchased from American Type Culture Collection were cultured in Dulbecco's modified Eagle's medium (DMEM; Sigma-Aldrich Co.) containing 10\% fetal bovine serum $(\mathrm{FBS})$ at $37^{\circ} \mathrm{C}$ in an atmosphere containing $5 \% \mathrm{CO}_{2}$.

\subsection{PPAR $\gamma$ activation monitored by luciferase reporter as-} say

The plasmids pM-PPAR $\gamma$, expressing a chimeric protein comprising a human-PPAR $\gamma$ ligand-binding domain and a DNA-binding domain of a yeast-derived GAL4 transcription factor, p $4 \times$ UASg-tk-luc, containing firefly luciferase gene linked downstream from a GAL4 responsive elements, and pRL-CMV, expressing Renilla luciferase under the control of CMV promoter were gift from Pharma Foods International Co., Ltd. CV-1 cells seeded in 100-mm Petri dishes were co-transfected with $2 \mu \mathrm{g}$ of pM-PPAR $\gamma, 4 \mu \mathrm{g}$ of $\mathrm{p} 4 \times$ UASg-tk-luc, and $0.04 \mu \mathrm{g}$ of pRL-CMV using Lipofectamine Reagent(Invitrogen, CA, USA). After $3.5 \mathrm{~h}$ incubation, the transfected cells were transferred to 96 -well plates at a density of $5 \times 10^{3}$ cells per well, and test samples, which were diluted in DMEM containing 4\% FBS to twice, were then treated to the cells and incubated for $24 \mathrm{~h}$.

Firefly and Renilla luciferase activities were measured using a Dual-Luciferase(R) Reporter Assay System (Promega, WI, USA), according to manufacturer's instructions. Luminescence intensity was measured for $10 \mathrm{~s}$ using a luminometer (Micro Lumat Plus; Berthold Japan Co., Ltd.), and luminescence intensity of firefly luciferase was normalized to that of Renilla luciferase. PPAR $\gamma$-activity was presented as percentages of the control group.

\subsection{PPAR $\gamma$ activation assays of normal-phase column fractions}

PPAR $\gamma$ activation assays were performed with seven normal-phase column fractions to investigate PPARy ligand activity. In these experiments, $100-$ and $50-\mu \mathrm{g} / \mathrm{mL}$ fractions were used and PPAR $\gamma$ ligand activities of fractions were expressed relative to that of controls (DMSO).

\subsection{Reversed-phase column chromatography and PPAR $\gamma$ activation assays of eluates}

Fraction 1 from normal-phase column chromatography was subjected to reverse-phase chromatography to prepare five reverse-phase column fractions. Reversed-phase chromatography was performed using a chromatographic glass column (length, $40 \mathrm{~cm}$; diameter, $2.2 \mathrm{~cm}$; volume, $152 \mathrm{~mL}$ ) packed with $50 \mathrm{~g}$ of ODS silica gel (YMC*GEL ODS-A $6 \mathrm{~nm}$ S-150 $\mu \mathrm{m}$; YMC Co., Ltd.) suspended in methanol. Subsequently, $100 \mathrm{~mL}$ of $100 \%$ degassed methanol was injected to prevent foaming and the packing material was equilibrated with 10 volumes $(760 \mathrm{~mL})$ of $10 \%$ aqueous degassed methanol. Fraction 1 $(117 \mathrm{mg})$ from normal-phase fractions was dissolved in methanol and added to the material. Aqueous solutions of degassed 10\%, 20\%, 30\%, 40\% and $100 \%(\mathrm{v} / \mathrm{v})$ methanol were injected $(180 \mathrm{~mL}$ each) onto the packing material in that order. Eluted fractions were then divided according to solvent compositions and were recovered (fractions 1-1-1-5). Fractions were then concentrated under reduced pressure and portions of each fraction were 
dissolved in DMSO. Separation of recovered fractions was confirmed using HPLC with a YMC-Pack ODS-A column $(250 \times 4.6 \mathrm{~mm}$ I.D. S-5 $\mu \mathrm{m} 30 \mathrm{~nm}$; YMC Co., Ltd. $)$ and graduated mobile phase of $20 \%-70 \%(\mathrm{v} / \mathrm{v})$ aqueous methanol (flow rate, $1 \mathrm{~mL} / \mathrm{min}$; column temperature, $40^{\circ} \mathrm{C}$ ). The absorbance of elutes were monitored at $280 \mathrm{~nm}$ using a UV-Vis detector SPD-20A (Prominence Modular HPLC, Shimazu Co., Kyoto, Japan).

\subsection{PPAR $\gamma$ activity measurement of reversed-phase col- umn fraction}

PPAR $\gamma$ activation was determined for reversed-phase column fractions 1-1-1-5 and the normal-phase column fraction 1 at $100 \mu \mathrm{g} / \mathrm{mL}$. In these experiments, the PPAR $\gamma$ agonist troglitazone ( $1 \mu \mathrm{M}$; Wako Pure Chemical Industries, Ltd.) was used as a positive control.

\subsection{Fractionation using HPLC}

HPLC chromatograms of reversed-phase column fractions indicated plurality of specific peaks in fractions 4 and 5 and PPAR $\gamma$-activating effects were confirmed. To isolate active ingredients, fractions were further fractionated according to specific peak retention times using reversedphase HPLC (YMC-Pack ODS-A column; $250 \times 4.6 \mathrm{~mm}$ I.D., S- $5 \mu \mathrm{m}, 30 \mathrm{~nm}$ ) with a graduated mobile phase of $20 \%$ $-70 \%$ aqueous methanol (flow rate, $1 \mathrm{~mL} / \mathrm{min}$; column temperature, $40^{\circ} \mathrm{C}$ ), and elutes were monitored at $280 \mathrm{~nm}$ using a UV-Vis detector SPD-20A (Prominence Modular HPLC, Shimazu Co.).

\subsection{Structural determination of peak fractions}

Structural analyses of the fraction of peak 5 , which contained the active ingredients, were performed using $\mathrm{LC} /$ ESI-MS, ${ }^{1} \mathrm{H}-\mathrm{NMR},{ }^{13} \mathrm{C}-\mathrm{NMR}$, HMBC and HSQC as described previously ${ }^{12)}$ and $m / z$ values were graphed against detection intensity.

\subsection{Synthesis and characterization of synthetic fra- glide-1 (5H4PB)}

Synthetic fraglide-1 (5H4PB) was synthesized to confirm the PPAR $\gamma$-activating effects of the active ingredient from Kozu. In these experiments, $100 \mathrm{mg}$ of glyoxylic acid monohydrate and $150 \mathrm{mg}$ of morpholine were dispersed in $450 \mu \mathrm{L}$ of 1,4-dioxane and $55 \mu \mathrm{L}$ of water was added dropwise. To the mixture, $140 \mathrm{mg}$ of phenylacetaldehyde was added, was left to stand at room temperature for $1 \mathrm{~h}$ and was then heated to reflux for $24 \mathrm{~h}$. The resulting product was concentrated under reduced pressure, followed by extraction with $2.5 \mathrm{~mL}$ of diethyl ether. The extracted diethyl ether layer was dehydrated, dried by addition of anhydrous magnesium sulphate and was then concentrated under reduced pressure. The concentrate was dissolved in an acetone/chloroform mixture and was then recrystallized to obtain $140 \mathrm{mg}$ of 5H4PB. 5H4PB was subjected to gas chromatography, ${ }^{1} \mathrm{H}-\mathrm{NMR}$ and ${ }^{13} \mathrm{C}-\mathrm{NMR}$ to confirm its identity with the active ingredient from Kozu.

\subsection{Measurement of UCP-1 gene expression}

UCP-1 expression was determined as previously described $^{13,14)}$. Briefly, C3H10T1/2 mesenchymal cells $\left(1 \times 10^{4}\right.$ cells $/ \mathrm{mL}$ ) were added to 12 -well plates and cultured until confluence. The fraction of peak 5 (fraglide-1) was added (final concentration $100 \mu \mathrm{g} / \mathrm{mL}$ ) and DMSO was used as a control. After $24 \mathrm{~h}$, mRNA was recovered from cells using Sepasol RNA super G (Nacalai Tesque, Inc.) chloroform/isopropanol extraction according to the manufacturer's protocol and mRNA concentrations were determined using a Nano Drop instrument (Thermo Fischer Scientific Inc., MA, USA). Subsequently, cDNAs were synthesized using Oligo dT primer (Thermo Fisher Scientific Inc.) and Super script (II) reverse transcriptase (Thermo Fisher Scientific Inc.) according to the manufacturer's instructions. UCP-1 mRNA expression was then determined using SYBR Green (Toyobo Co., Ltd., Osaka, Japan) and a LightCycler (Roche Applied Science) with the following primers for murine UCP-1: forward, 5'-ggctctacgactcagtcca-3'; reverse, 5'-taagccggctgagatcttgt-3. PCR cycling conditions were as follows: $95^{\circ} \mathrm{C}$ for 5 min followed by 50 cycles of $95^{\circ} \mathrm{C}$ for 10 $\mathrm{s}, 60^{\circ} \mathrm{C}$ for $30 \mathrm{~s}$ and $72^{\circ} \mathrm{C}$ for $1 \mathrm{~s}$, and a final incubation at $40^{\circ} \mathrm{C}$ for $10 \mathrm{~s}$.

\subsection{Statistical analyses}

The data were presented as the mean \pm standard error of mean (SEM). All in vitro experiments were performed three times independently. Differences between control and treatment groups were examined by using Student's t-tests. Statistical analyses between multiple groups were performed by ANOVA. Statistical comparisons were made by Tukey-Kramer test. Differences with ${ }^{* *} p<0.01$ or $* p<$ 0.05 were considered significant.

\section{RESULTS}

\subsection{Isolation of PPAR $\gamma$-activating ingredients from aged vinegar}

Redundant lipid components were removed from Kozu by delipidation (Fig. 1A), and a butenolide compound (Fig. 3A) was effectively extracted using solvent extraction and fractionation treatments as described in the Methods section (Figs. $1 \mathrm{~A}$ and $\mathrm{B}$ ).

Following fractionation of concentrated chloroform extracts using normal-phase silica gel chromatography, seven fractions were obtained and PPAR $\gamma$ activation was determined ${ }^{15)}$. In these experiments, PPAR $\gamma$ activation was greatest in the presence of fraction 1 (mobile phase, benzene:acetone $=20: 1(\mathrm{v} / \mathrm{v}))$ and increased in a concentration-dependent manner (Fig. 1C). Five fractions were then 

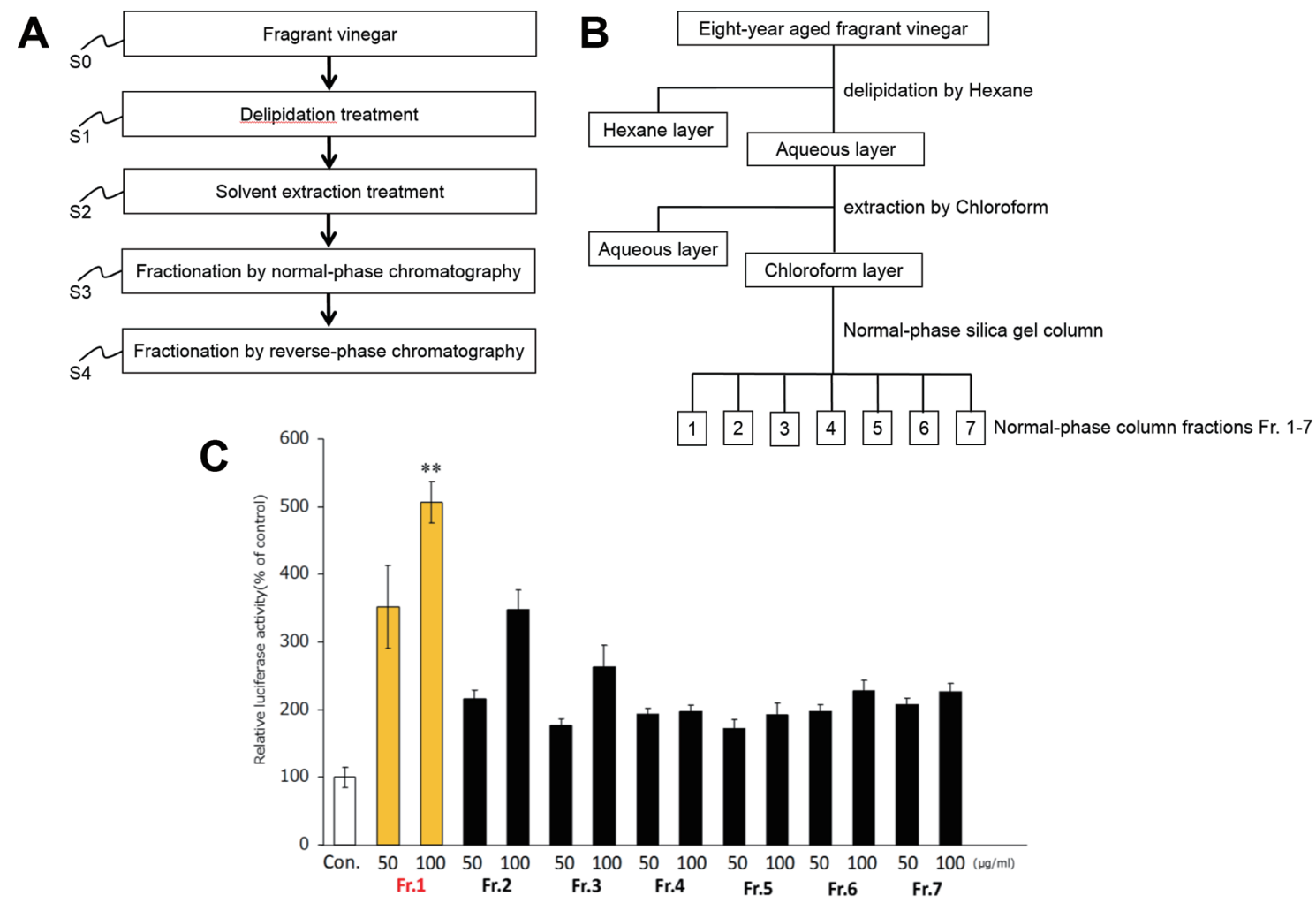

Fig. 1 Serial isolation of a peroxisome proliferation-activated receptor (PPAR) $-\gamma$ agonist from Kozu.

(A) Flowchart of protocols for isolating the butenolide ingredient (compound?) from Kozu according to PPAR $\gamma$ activation; (B) Schematic of preparation of normal-phase column fractions from Kozu; (C) PPARy ligand activities of normal-phase column fractions were determined using luciferase reporter assays of transcription from the target promoter for the mitochondrial uncoupling protein-1. Reporter activities were expressed relative to that of the vehicle control (100\%). Data in bar graphs were presented as means \pm standard error of mean(SEM) of 5 wells; * $p<$ 0.05 and $* * p<0.01$ vs. vehicle control.

eluted from reverse-phase chromatography using a silica gel modified with an octadecylsilyl (ODS) group $\left(\mathrm{C}_{18}\right)$ and a methanol-water gradient mobile phase (Fig. 2A). In subsequent reporter assays, PPAR $\gamma$ activation was greatest in the presence of fractions 1-4 (elution solvent, water:methanol $=60: 40$ ) and 1-5 (elution solvent, 100\% methanol; Fig. $2 \mathrm{~B}$ ), and peaks from the corresponding chromatogram (Fig. 2C) were used to identify elution times for active ingredients. Following the elution of fractions corresponding to peaks $2,3,5,6,8,10,15$ and 31 , PPAR $\gamma$ activation assays indicated that peak 5 contained the ingredient of interest (Fig. 2D). The high-performance liquid chromatography (HPLC) analysis of peak 5 showed a single peak and was found to be high purity (Fig. 2E).

In recent years, synthetic PPAR $\gamma$ agonists have been shown to convert white adipocytes that store fat into brown like (beige/brite) adipocytes that burn fat ${ }^{16)}$, in part by upregulating UCPs. Because UCPs are known targets of PPARs, UCP-1 mRNA expression level was analyzed to confirm that the fraglide-1 as a PPAR $\gamma$ agonist might lead to conversion of white adipocytes to brown like(beige/ brite) adipocytes (Fig. 2F). In these experiments, UCP-1 expression was dramatically elevated in the presence of the peak 5 fraction. Therefore, it was speculated that the compound in peak 5 fraction can enhance heat production from lipid catabolism.

\subsection{Structural determination of the active ingredient}

The results of liquid chromatography coupled with electro spray ionization - mass spectrometry (LC/ESI-MS) are shown in Fig. 3B with $\mathrm{m} / z$ values and corresponding detection intensities. These analyses show that peak 5 has a molecular weight of $176.0473 \mathrm{~g} / \mathrm{mol}$, which corresponds with the rational formula $\mathrm{C}_{10} \mathrm{H}_{8} \mathrm{O}_{3}$. The mass spectrum of 351.0870 observed in Fig. $3 \mathrm{C}$ might be because a 5H4PB dimer was formed by non-covalent electrostatic interaction such as ionic or hydrogen bonding. Subsequent proton nuclear magnetic resonance $\left({ }^{1} \mathrm{H}-\mathrm{NMR}\right),{ }^{13} \mathrm{C}$ nuclear magnetic resonance $\left({ }^{13} \mathrm{C}-\mathrm{NMR}\right),{ }^{1} \mathrm{H}$-detected multi-bond heteronuclear multiple quantum coherence spectrum (HMBC) and ${ }^{1} \mathrm{H}$-detected multiple quantum coherence spectrum (HSQC) analyses were performed as previously de- 
A

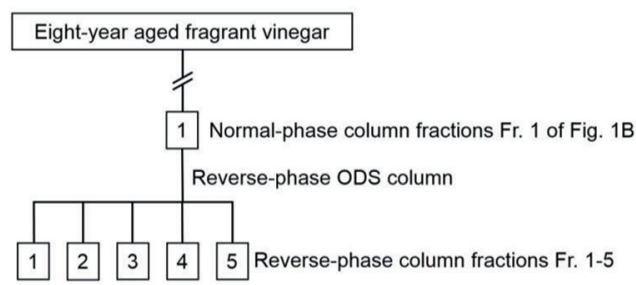

C

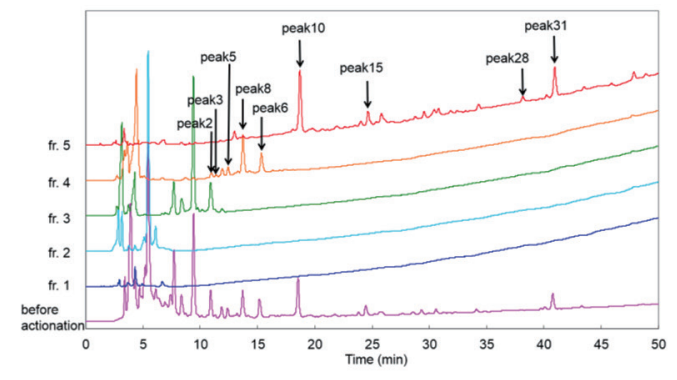

E

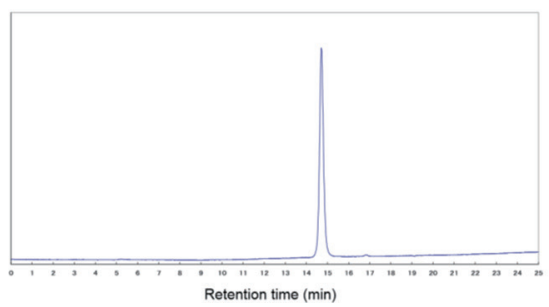

B
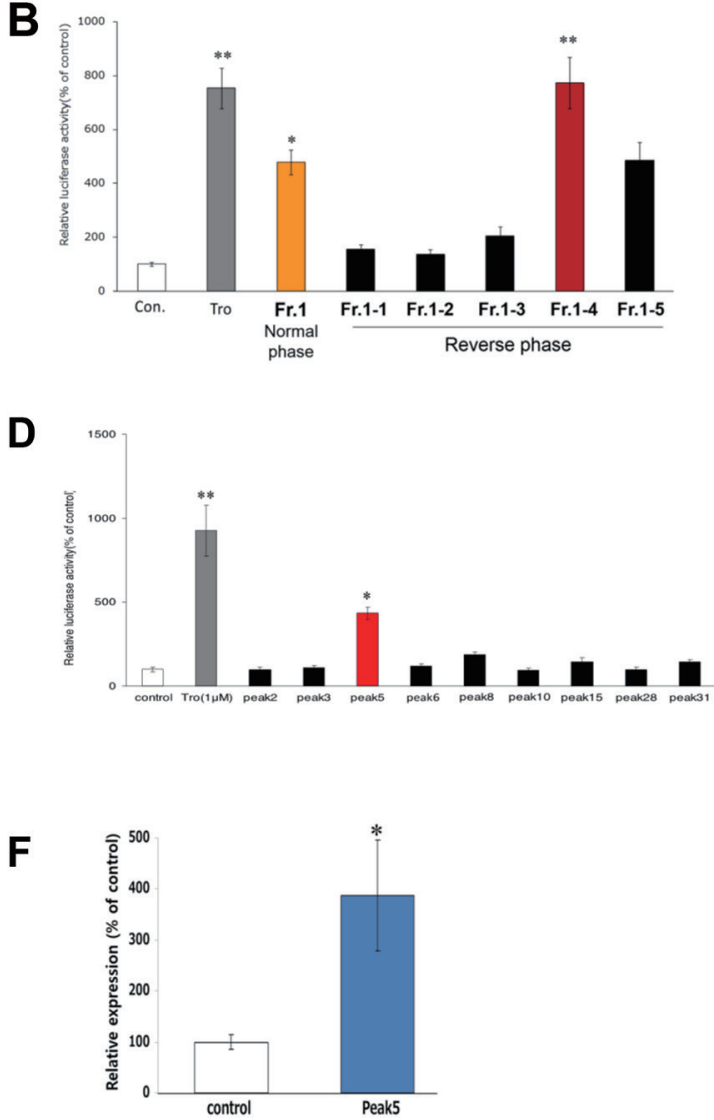

Fig. 2 Isolation of PPAR $\gamma$-activating ingredients from Kozu.

(A) A flowchart of methods for preparing reversed-phase column fractions; (B) PPAR $\gamma$ ligand activity of reversedphase column fractions; the ordinate of the drawing shows the ratio of the activity of the fraction to the activity of the control ingredient (troglitazone, Tro). (C) Chromatogram from high-performance liquid chromatography analysis of reverse-phase column fractions (fractions 1-1-1-5); the abscissa was shown retention time(min). (D) PPAR $\gamma$ ligand activity of peak fractions. (E) Chromatogram from high-performance liquid chromatography analysis of peak 5; the ordinate was shown detection intensity and the abscissa shows retention time ( $\min$ ). (F) Expression of uncoupling protein (UCP) -1 in the presence of the fraction of peak 5 . Expression levels and luciferase activities were expressed relative to those of the vehicle control $(100 \%)$. Data in bar graphs were presented as means \pm SEM of 5 well; $* p<0.05$ and ${ }^{* *} p<0.01$ vs. vehicle control.

scribed $^{12)}$. The structure of the compound in peak 5 was analyzed with ${ }^{1} \mathrm{H}$ NMR and ${ }^{13} \mathrm{C}$ NMR. The ${ }^{1} \mathrm{H}-\mathrm{NMR}$ spectrum of the compound in peak 5 showed as follows $(500 \mathrm{MHz}$, CD3OD) : $\delta 7.80-7.78\left(2 \mathrm{H}, \mathrm{d}, \mathrm{J}=6.8, H-2^{\prime} \mathrm{A}, H-2^{\prime} \mathrm{B}\right), 7.50-$ $7.45\left(3 \mathrm{H}, \mathrm{m}, H\right.$-3'A, $\left.H-3^{\prime} \mathrm{B}, H-4^{\prime}\right)$ assigned to benzene protons, $6.59(1 \mathrm{H}, \mathrm{s}, H-5)$ assigned to olefin proton, 6.53 $(1 \mathrm{H}, \mathrm{s}, H-3)$ assigned to a methine proton. The ${ }^{13} \mathrm{C}-\mathrm{NMR}$ spectrum of the compound in peak 5 showed as follows $\delta$ (125 MHz, CD3OD): 173.4 (C-2) assigned to carbonyl carbon, $165.4(C-4)$ assigned to phenyl substituted doublebond carbon, $132.5\left(C-4^{\prime}\right), 131.0\left(C-1^{\prime}\right), 130.0\left(C-2^{\prime} \mathrm{A}, C-2^{\prime}\right.$ B), $129.2\left(C-3^{\prime} \mathrm{A}\right.$ and $\left.C-3^{\prime} \mathrm{B}\right)$ assigned to benzene carbons , $115.6(C-3)$ assigned to methylene carbon, $100.0(C-5)$ assigned to a hydroxyl-substituted carbon. These analyses suggested that the structure of the component in peak 5 was 5-hydroxy-4-phenyl-butenolide (fraglide-1; Fig. 3A). It was predicted that this ingredient was present in Kozu at $0.54 \mu \mathrm{g} / \mathrm{mL}$ using elute of $5 \mathrm{H} 4 \mathrm{~PB}$ from HPLC as a standard (data not shown).

Luciferase reporter assays confirmed that 5H4PB (Fig. 3C) (Tokyo Chemical Industry Co., Ltd., Tokyo, Japan) enhanced PPAR $\gamma$ ligand binding activity dose-dependently (Fig. 4).

\section{DISCUSSION}

Aged vinegar has a long history of use as a traditional Chinese medicine and various vinegars have been shown to ameliorate obesity and associated metabolic disorders in 
A
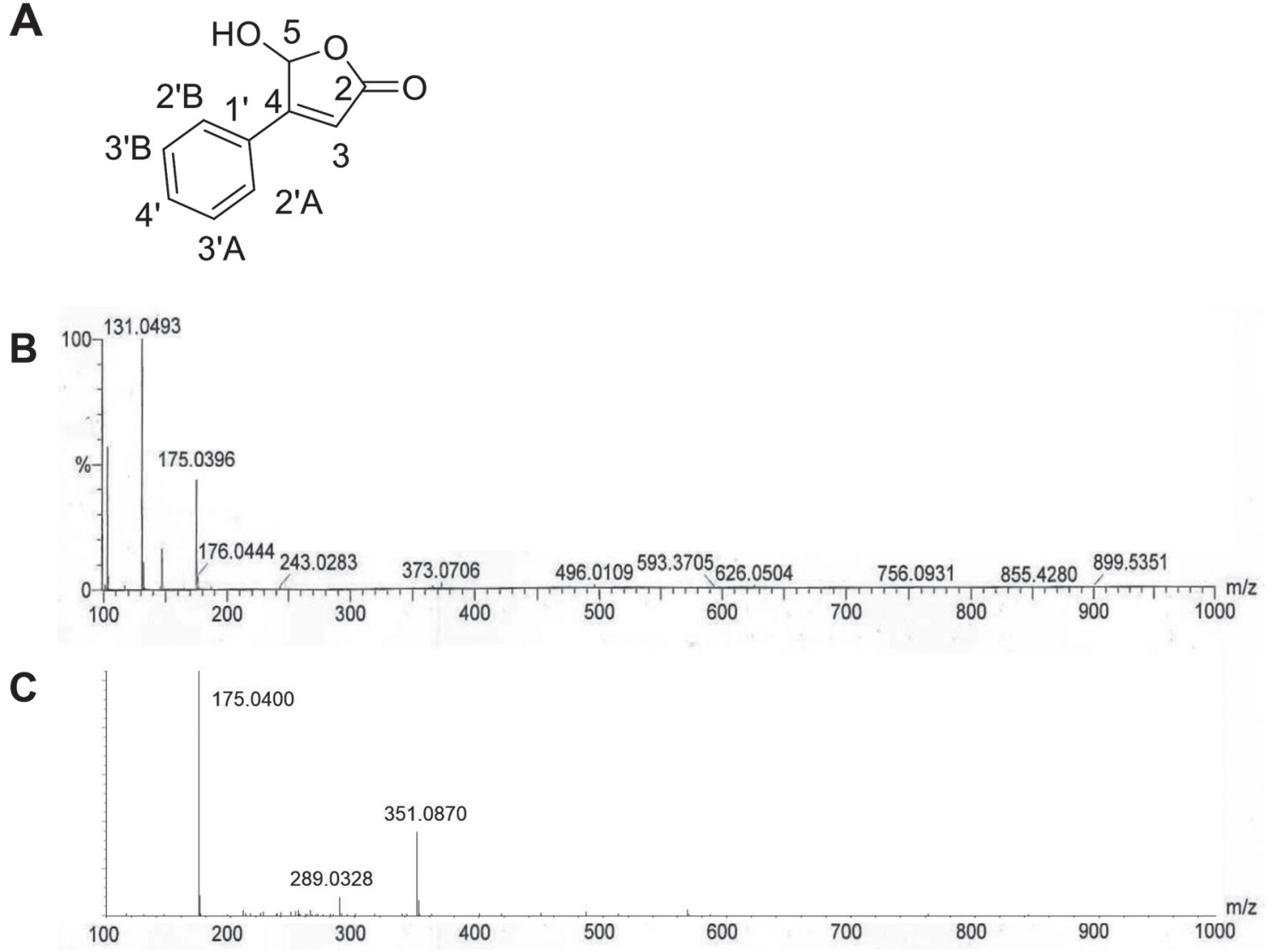

Fig. 3 Structural determination of the active ingredient.

(A) The chemical structure of 5-hydroxy-4-phenyl-butenolide (fraglide-1); (B) Liquid chromatography coupled with tandem mass spectrometry for the fraction of peak 5; the ordinate was shown detection intensity and the abscissa was shown $\mathrm{m} / z$ values; (C) Liquid chromatography coupled with tandem mass spectrometry for synthetic fraglide-1 (5H4PB).

multiple studies ${ }^{2,17-20)}$. However, although the contents of aged Chinese vinegars have been characterized ${ }^{1,21,22)}$ and fermentation products have been described ${ }^{23-25)}$, no active ingredient has been identified before and only anecdotal evidence of the health benefits of Kozu is available. In a recent study, Yusoff et al. demonstrated antihyperglycaemic effects of extracts from Nypa fruticans Wurmb. vinegar and described mechanisms that led to inhibition of glucose transporters ${ }^{19)}$. Similarly, these authors showed anti-diabetic and anti-oxidant properties of this Malaysian vinegar, but did not identify or isolate active ingredients ${ }^{18)}$. In addition, Kondo et al. associated acetic acid consumption with lowered anthropometric parameters in a randomized placebo controlled trial ${ }^{2)}$. However, in a recent review of the benefits of vinegar consumption, limited evidence is reported for the use of vinegars as adjuvant therapies for diabetes ${ }^{17)}$. In contrast with regular vinegars, the present Kozu vinegar contains multiple organic ingredients that are products of long fermentation and aging times. With traditional expectations of anti-obesity effects of Kozu, it follows that the unidentified active ingredients may include ingredients that effect energy metabolic pathways.

PPARs have been identified as ligand-dependent intranuclear transcription factors that regulate glucose and lipid metabolism and have been considered as targets for pharmaceutical anti-obesity interventions ${ }^{26)}$. Furthermore, UCP-1 generates heat by leaking proton across the mitochondria inner membrane, thus uncoupling oxidative phosphorylation without ATP production ${ }^{13,14)}$. Moreover, UCP-1 is specifically expressed in brown adipocytes, and a recent study showed that particular synthetic PPAR $\gamma$ agonists convert white fat storing cells into brown like(beige/brite) fat cells ${ }^{16)}$. Thus, PPAR $\gamma$ remains an important target for agents that may ameliorate metabolic disorders and obesity and offers a potential mechanism for the anti-obesity effects of aged vinegar. Accordingly, previous studies have demonstrated the presence of PPAR $\gamma$ agonists in other natural preparations ${ }^{10,27-30)}$. In addition, an anti-obesity ingredient that acts on UCP-1 was found in edible seaweed, warranting further investigations of the anti-diabetic effects of natural ingredients from various herbs and edible plants $^{10,31,32)}$. 


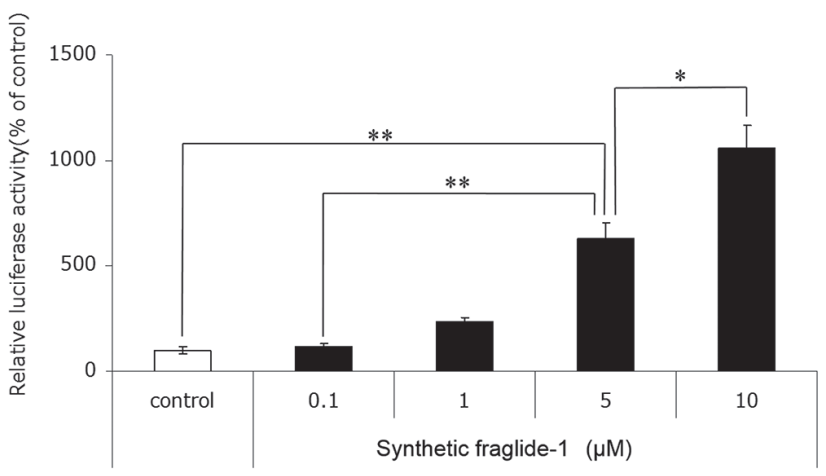

Fig. 4 PPAR $\gamma$ ligand activity by synthetic fraglide-1 (5H4PB).

PPAR $\gamma$ ligand activity of $5 \mathrm{H} 4 \mathrm{~PB} .{ }^{*} p<0.05$ and ${ }^{* *} p$ $<0.01$. Data in bar graphs were presented as means \pm SEM of 5 well.

In the present study, serial fractionation of the aged vinegar Kozu was performed based on PPAR $\gamma$ activation assays using Cell to identify fractions containing active ingredients. These procedures and corresponding PPAR $\gamma$ activity assays revealed the presence of a potent agonist in chromatographic fractions of Kozu extracts. Additionally, subsequent spectral analyses identified the butenolide compound, fraglide-1. In further experiments with 5H4PB, luciferase reporter expression from the PPAR $\gamma$ promoterbinding region was increased dose-dependently (Fig. 4), and specific activity was concentration dependent up to 10 $\mu \mathrm{M}$. Moreover, subsequent experiments confirmed that fraglide- 1 is an agonist for PPAR $\gamma$ and it also increased expression of the target gene UCP-1. Taken together, these data indicate the presence of a naturally occurring PPARy agonist in Kozu that may offer an alternative anti-diabetes drug to thiazolidinediones such as troglitazone.

In summary, the present lower alkanol extract of Kozu exhibited PPAR $\gamma$-activating effects that may improve sugar metabolism by stimulating fatty acid oxidation, particularly via the actions of mitochondrial UCP-1.

In conclusion, it was shown that the active ingredient "Fraglide-1" from Kozu was successfully found as a new agonist for PPAR $\gamma$ at cellular level. Although animal tests and clinical studies are undergoing, it is highly possible that the Fraglide-1 is a drug-lead compound or a dietary supplement for amelioration of pathological conditions that are associated with PPAR $\gamma$, such as insulin resistance, diabetes mellitus, obesity, hyperlipidemia, and fatty liver and metabolic syndrome.

\section{References}

1) Xiao, Z.; Dai, S.; Niu, Y.; Yu, H.; Zhu, J.; Tian, H.; Gu, Y. Discrimination of Chinese vinegars based on head- space solid-phase microextraction-gas chromatography mass spectrometry of volatile compounds and multivariate analysis. J. Food. Sci. 76, C1125-1135 (2011).

2) Kondo, T.; Kishi, M.; Fushimi, T.; Ugajin, S.; Kaga, T. Vinegar intake reduces body weight, body fat mass, and serum triglyceride levels in obese Japanese subjects. Biosci. Biotechnol. Biochem. 73, 1837-1843 (2009).

3) Ginter, E.; Simko, V. Type 2 diabetes mellitus, pandemic in 21st century. Adv. Exp. Med. Biol. 771, 42-50 (2012).

4) Porras, A.; Valladares, A.; Alvarez, A.M.; Roncero, C.; Benito, M. Differential role of PPAR gamma in the regulation of UCP-1 and adipogenesis by TNF-alpha in brown adipocytes. FEBS Lett. 520, 58-62(2002).

5) Xu, H.; Barnes, G.T.; Yang, Q.; Tan, G.; Yang, D.; Chou, C.J.; Sole, J.; Nichols, A.; Ross, J.S.; Tartaglia, L.A.; Chen, H. Chronic inflammation in fat plays a crucial role in the development of obesity-related insulin resistance. J. Clin. Invest. 112, 1821-1830(2003).

6) Stulc, T.; Ceska, R. Rosiglitazone in the prevention of diabetes and cardiovascular disease: dream or reality? Med. Sci. Monit. 14, RA45-47(2008).

7) Akazawa, S.; Sun, F.; Ito, M.; Kawasaki, E.; Eguchi, K. Efficacy of troglitazone on body fat distribution in type 2 diabetes. Diabetes Care 23, 1067-1071 (2000).

8) Yu, Y.H.; Wu, S.C.; Cheng, W.T.; Mersmann, H.J.; Shen, T.L.; Ding, S.T. The function of porcine PPAR $\gamma$ and dietary fish oil effect on the expression of lipid and glucose metabolism related genes. J. Nutr. Biochem. 22, 179-186 (2011).

9) Matsumura, T.; Kinoshita, H.; Ishii, N.; Fukuda, K.; Motoshima, H.; Senokuchi, T.; Taketa, K.; Kawasaki, S.; Nishimaki-Mogami, T.; Kawada, T.; Nishikawa, T.; Araki, E. Telmisartan exerts antiatherosclerotic effects by activating peroxisome proliferator-activated receptorgamma in macrophages. Arterioscler. Thromb. Vasc. Biol. 31, 1268-1275 (2011).

10) Hosokawa, M.; Kudo, M.; Maeda, H.; Kohno, H.; Tanaka, T.; Miyashita, K. Fucoxanthin induces apoptosis and enhances the antiproliferative effect of the PPAR $\gamma$ ligand, troglitazone, on colon cancer cells. Biochim. Biophys. Acta. 1675, 113-119(2004).

11) Brietzke, S.A. Oral antihyperglycemic treatment options for type 2 diabetes mellitus. Med. Clin. North. Am. 99, 87-106 (2015).

12) Koshino, H.; Yoshihara, T.; Okuno, M.; Sakamura, S.; Tajimi, A.; Shimanuki, T. Gamahonolides A, B, and gamahorin, novel antifungal compounds from stromata of Epichloe typhina on Phleum pratense. Biosci. Biotechnol. Biochem. 56, 1096-1099(1992).

13) Sakamoto, T.; Takahashi, N.; Sawaragi, Y.; Naknukool, S.; Yu, R.; Goto, T.; Kawada, T. Inflammation induced 
by RAW macrophages suppresses UCP1 mRNA induction via ERK activation in 10T1/2 adipocytes. Am. J. Physiol. Cell Physiol. 302 (2), C463-472 (2012).

14) Rousset, S.; Alves-Guerra, M.C.; Mozo, J.; Miroux, B.; Cassard-Doulcier, A.M.; Bouilland, F.; Ricquier, D. The biology of mitochondrial uncoupling proteins. Diabetes 53 (Suppl. 1), S130-135(2004).

15) Nagai, H.; Ebisu, S.; Abe, R.; Goto, T.; Takahashi, N.; Hosaka, T.; Kawada, T. Development of a novel PPAR ligand screening system using pinpoint fluorescenceprobed protein. Biosci. Biotechnol. Biochem. 75, 337-341 (2011).

16) Ohno, H.; Shinoda, K.; Spiegelman, B.M.; Kajimura, S. PPARgamma agonists induce a white-to-brown fat conversion through stabilization of PRDM16 protein. Cell Metab. 15, 395-404(2012).

17) Kohn, J.B. Is vinegar an effective treatment for glycemic control or weight loss? J. Acad. Nutr. Diet. 115, 1188 (2015).

18) Yusoff. N.A.; Yam, M.F.; Ben, H.K.; Abdul Razak, K.N.; Widyawati, T.; Mahmud, R.; Ahmad, M.; Asmawi, M.Z. Antidiabetic and antioxidant activities of Nypa fruticans Wurmb. vinegar sample from Malaysia. Asian Pac. J. Trop. Med. 8, 595-605(2015).

19) Yusoff, N.A.; Ahmad, M.; Al-Hindi, B.; Widyawati, T.; Yam, M.F.; Mahmud, R.; Abdul Razak, K.N.; Asmawi, M.Z. Aqueous extract of Nypa fruticans Wurmb. Vinegar alleviates postprandial hyperglycemia in normoglycemic rats. Nutrients 7, 7012-7026 (2015).

20) Setorki, M.; Asgary, S.; Eidi, A.; Rohani, A.H.; Khazaei, M. Acute effects of vinegar intake on some biochemical risk factors of atherosclerosis in hypercholesterolemic rabbits. Lipids Health Dis. 9, 10 (2010).

21) Chen, Q.; Ding, J.; Cai, J.; Sun, Z.; Zhao, J. Simultaneous measurement of total acid content and soluble salt-free solids content in Chinese vinegar using nearinfrared spectroscopy. J. Food. Sci. 77, C222-227 (2012).

22) Wang, C.Y.; Zhang, J.; Gui, Z.Z. Acetobacter bacteria are found in Zhenjiang vinegar grains. Genet. Mol. Res. 14, 5054-5064 (2015).

23) Xu, W.; Xu, Q.P.; Chen, J.H.; Lu, Z.M.; Xia, R.; Li, G.Q.; $\mathrm{Xu}, \mathrm{Z} . \mathrm{H}$;; Ma, Y.H. Ligustrazine formation in Zhenjiang aromatic vinegar: changes during fermentation and storing process. J. Sci. Food. Agric. 91, 1612-1617 (2011).
24) Wang, Z.M.; Lu, Z.M.; Yu, Y. J.; Li, G.Q.; Shi, J.S.; Xu, Z.H. Batch-to-batch uniformity of bacterial community succession and flavor formation in the fermentation of Zhenjiang aromatic vinegar. Food Microbiol. 50, 64-69 (2015).

25) Xu, W.; Huang, Z.; Zhang, X.; Li, Q.; Lu, Z.; Shi, J.; Xu, $\mathrm{Z}$; Ma, Y. Monitoring the microbial community during solid-state acetic acid fermentation of Zhenjiang aromatic vinegar. Food Microbiol. 28, 1175-1181 (2011).

26) Mansour, M. The roles of peroxisome proliferator-activated receptors in the metabolic syndrome. Prog. Mol. Biol. Transl. Sci. 121, 217-266 (2014).

27) Goto, T.; Nagai, H.; Egawa, K.; Kim, Y.; Kato, S.; Taimatsu, A.; Sakamoto, T.; Ebisu, S.; Hohsaka, T.; Miyagawa, H.; Murakami, S.; Takahashi, N.; Kawada, T. Farnesyl pyrophosphate regulates adipocyte functions as an endogenous PPARgamma agonist. Biochem. J. 438, 111-119 (2011).

28) Takahashi, N.; Yao, R.; Kang, M. S.; Senda, M.; Ando, C.; Nishimura, K.; Goto, T.; Hirai, S.; Ezaki, Y.; Kawada, T. Dehydroabietic acid activates peroxisome proliferatoractivated receptor-gamma and stimulates insulin-dependent glucose uptake into 3T3-L1 adipocytes. Biofactors 37, 309-314 (2011).

29) Goto, T.; Takahashi, N.; Kato, S.; Kim, Y. I.; Kusudo, T.; Taimatsu, A.; Egawa, K.; Kang, M. S.; Hiramatsu, T.; Sakamoto, T.; Uemura, T.; Hirai, S.; Kobayashi, M.; Horio, F.; Kawada, T. Bixin activates PPARalpha and improves obesity-induced abnormalities of carbohydrate and lipid metabolism in mice. J. Agric. Food Chem. 60, 11952-11958(2012).

30) Takahashi, N.; Goto, T.; Taimatsu, A.; Egawa, K.; Katoh, S.; Kusudo, T.; Sakamoto, T.; Ohyane, C.; Lee, J. Y.; Kim, Y. I.; Uemura, T.; Hirai, S.; Kawada, T. Bixin regulates mRNA expression involved in adipogenesis and enhances insulin sensitivity in 3T3-L1 adipocytes through PPARgamma activation. Biochem. Biophys. Res. Commun. 390, 1372-1376 (2009).

31) Maeda, H.; Hosokawa, M.; Sashima, T.; Miyashita, K. Antiobesity effect of fucoxanthin from edible seaweeds and is multibiological functions. Functional Food and Health 993, 376-388 (2008).

32) Gammone, M.A.; D'Orazio, N. Anti-obesity activity of the marine carotenoid fucoxanthin. Mar. Drugs. 13, 2196-2214(2015). 